\title{
Acurácia do diagnóstico nutricional de pomares de mangueiras obtido por três fórmulas DRIS
}

\author{
Paulo Guilherme Salvador Wadt(1) e Davi José Silva(2) \\ (1)Embrapa Acre, Caixa Postal 321, CEP 69908-970 Rio Branco, AC. E-mail: paulo@cpafac.embrapa.br (2)Embrapa Semiarido, Caixa Postal 23, \\ CEP 56302-970 Petrolina, PE. E-mail: davi@cpatsa.embrapa.br.
}

\begin{abstract}
Resumo - O objetivo deste trabalho foi testar a aplicação de medidas de acurácia para determinar a utilidade de diagnósticos nutricionais para comparação de fórmulas DRIS, e avaliar o emprego dessas medidas no processo de modelagem das funções do Sistema Integrado de Diagnose e Recomendação (DRIS). Foram avaliadas três fórmulas: relações bivariadas simplificadas; relações multivariadas; e modelagem das funções DRIS. Foram utilizados dados de monitoramento nutricional de pomares de mangueiras cultivadas no Vale do São Francisco. O desempenho das fórmulas foi avaliado com base na concordância de seus diagnósticos com o proporcionado pelo método das faixas de suficiência. Foram determinadas as acurácias global e de insuficiência, de equilíbrio, de excesso, de deficiência, de suficiência e de toxicidade nutricional. A fórmula simplificada e a de relações multivariadas tiveram resultados semelhantes para as acurácias de deficiência e insuficiência, mas inferior ao da fórmula DRIS para modelagem das funções. Esta última, por possibilitar o ajuste do coeficiente de sensibilidade de cada função DRIS, mostrou-se promissora para diminuir riscos de diagnósticos falsos de deficiência (deixar de recomendar o nutriente quando ele é necessário) e de insuficiência (recomendar o nutriente quando ele não é necessário). No entanto, a fórmula DRIS para modelagem das funções não mostra desempenho satisfatório nas demais medidas de acurácia.
\end{abstract}

Termos para indexação: Mangifera indica, faixas de suficiência, diagnose foliar, potencial de resposta.

\section{Nutritional diagnosis accuracy of mango tree orchards through three DRIS formulas}

\begin{abstract}
The objective of this work was to test the application of accuracy measures to determine the usefulness of nutritional diagnosis to compare different DRIS formulas, and to evaluate the use of these measures in the DRIS function modeling process. Three DRIS formulas were evaluated: bivariate relations (simplified); multivariate relations; and DRIS function modeling. Monitoring data from commercial mango orchards grown at São Francisco River Valley, Brazil, were used. Formula performances were evaluated based on the agreement of their diagnoses with the one provided by the sufficiency range method. Global accuracy, and the ones for insufficiency, equilibrium, excess, deficiency, sufficiency and nutritional toxicity were determined. The simplified and multivariate formulas showed similar results for insufficiency and deficiency accuracies, but they had a lower performance than the DRIS function modeling formula. This latter, for allowing of the adjustment of each DRIS function sensibility coefficient, showed to be promising in reducing the risks of false diagnosis for deficiency (as for not recommend the nutrient when it is necessary) and for insufficiency (as for recommend the nutrient when it is not necessary). However, the DRIS formula for function modeling does not show a satisfactory performance for other accuracy measures.
\end{abstract}

Index terms: Mangifera indica, sufficiency ranges, foliar diagnosis, response potential.

\section{Introdução}

A utilidade de metodologias de avaliação do estado nutricional das plantas depende da capacidade em identificar corretamente qual nutriente limita a produtividade das culturas, de modo a possibilitar a correção de desequilíbrios nutricionais, pela correção no manejo da adubação (Beverly \& Hallmark, 1992).

$\mathrm{Na}$ literatura, diferentes fórmulas DRIS têm sido propostas como alternativas para a obtenção de diagnósticos nutricionais úteis, tais como a fórmula simplificada (Jones, 1981), em que o equilíbrio nutricional é determinado com base exclusivamente na medida padronizada do desvio de uma relação bivariada, em relação a um valor ótimo, ou à fórmula para relação multivariada (Parent \& Dafir, 1992), que se fundamenta em relações log-transformadas entre cada nutriente e a média geométrica da composição nutricional do tecido amostrado. Entretanto, é comum que se opte por determinada fórmula sem realizar comparações entre elas. 
Para validação do diagnóstico nutricional, as frequências de ocorrência dos nutrientes, nas diferentes classes de interpretação do estado nutricional (Wadt \& Novais, 1999), são comparadas com as frequências esperadas na hipótese de que o diagnóstico fosse aleatório. Em estudos mais detalhados, o número de diagnósticos concordantes entre diferentes métodos é computado (Partelli et al., 2006). Entretanto, nenhuma dessas alternativas avalia objetivamente a utilidade dos diagnósticos obtidos, já que elas não possibilitam identificar o grau de acerto para os diferentes tipos de diagnósticos possíveis (Wadt \& Lemos, 2010).

O primeiro método proposto para avaliar metodicamente a utilidade de sistemas de diagnóstico do estado nutricional foi o sistema "prescient diagnostic analysis methodogy" (Beverly \& Hallmark, 1992). Mais recentemente, com base no trabalho desses autores, Wadt \& Lemos (2010) propuseram sete medidas de acurácia para tornar essa avaliação compatível com a utilização do critério do potencial de resposta à adubação (PRA), proposto por Wadt (2005), em que a acurácia foi definida como a medida da proximidade entre o valor obtido em um diagnóstico nutricional e o verdadeiro estado nutricional da planta.

Além de avaliarem a utilidade do diagnóstico nutricional, as medidas de acurácia podem ser utilizadas para a modelagem das fórmulas DRIS. Bataglia et al. (2004) testaram, com pouco sucesso, diferentes combinações pré-estabelecidas para o fator de ajuste (coeficiente de sensibilidade - fk), com o objetivo de obter melhoria na qualidade dos diagnósticos. Entretanto, Wadt et al. (2007) sugerem que as funções DRIS podem ser definidas para cada tipo de nutriente, e que o valor mais adequado para o fator de ajuste, a ser atribuído a cada função DRIS, pode ser determinado por meio de procedimentos iterativos. Nesses procedimentos se mensura o desempenho do diagnóstico nutricional e se ajusta o valor do fator $\mathrm{k}$, até que se consiga melhorar o desempenho do diagnóstico nutricional a um determinado grau de acerto pré-estabelecido (Wadt \& Lemos, 2010).

O objetivo deste trabalho foi testar a aplicação de medidas de acurácia para determinar a utilidade dos diagnósticos nutricionais na comparação de fórmulas
DRIS, e avaliar o uso dessas medidas no processo de modelagem das funções DRIS.

\section{Material e Métodos}

Os dados de campo foram obtidos de lavouras comerciais de manga (Mangifera indica L.), variedade Tommy Atkins, cultivadas sob irrigação, e referem-se ao mesmo conjunto de dados utilizados no trabalho de Wadt et al. (2007). Foram avaliados, mensalmente, durante dois anos (1988 e 1999), dez pomares localizados em propriedades agrícolas do Submédio São Francisco, na região semiárida do Nordeste do Brasil, no total de 145 amostras de pomares comerciais. Folhas maduras, inteiras e sadias, preferencialmente na penúltima brotação ou na brotação terminal, foram coletadas em número de quatro por planta, nas posições referentes aos pontos cardeais, na altura mediana da planta, em 25 árvores por área (Silva et al., 2002). O material vegetal coletado nas amostragens foi acondicionado em sacos de papel e transportado para o laboratório. Foram determinadas as concentrações de N, P, K, Ca e $\mathrm{Mg}\left(\mathrm{g} \mathrm{kg}^{-1}\right)$, e de B, Fe, Mn, $\mathrm{Zn} \mathrm{e} \mathrm{Cu}\left(\mathrm{mg} \mathrm{kg}^{-1}\right)$.

As normas DRIS foram obtidas de todo o conjunto de dados de monitoramento, sem a divisão das amostras em classes de produtividade, em razão do pequeno número de lavouras avaliadas. Este procedimento, na obtenção de normas DRIS sem transformação logarítmica, indicou que as normas geradas por essa metodologia foram estatisticamente semelhantes às obtidas por Pinto (2002), para a maioria das relações nutricionais (Wadt et al., 2007). As médias e variâncias das relações bivariadas transformadas logaritmicamente e das relações multivariadas também foram obtidas do conjunto de dados de monitoramento, conforme Beverly \& Hallmark (1992) e Parent \& Dafir (1992), respectivamente. A exceção foi que, nas relações bivariadas, a variância foi calculada para cada relação, e não somente para a concentração do nutriente, conforme proposto originalmente por Beverly \& Hallmark (1992).

Os índices DRIS foram calculados a partir das normas bivariadas, e os índices DRIS de cada nutriente (In) foram obtidos com uso de todas as relações entre dois nutrientes, na forma direta e inversa: 
In $=\left[\mathrm{f}\left(\mathrm{A} / \mathrm{B}_{1}\right)+\mathrm{f}\left(\mathrm{A} / \mathrm{B}_{2}\right)+\ldots+\mathrm{f}\left(\mathrm{A} / \mathrm{B}_{\mathrm{n}}\right)-\mathrm{f}\left(\mathrm{B}_{1} / \mathrm{A}\right)-\right.$ $\left.f\left(B_{2} / A\right)-\ldots-f\left(B_{n} / A\right)\right] /(2 n)$,

em que: In corresponde ao índice DRIS de um dado nutriente; $\mathrm{f}(\mathrm{A} / \mathrm{B})$ corresponde à função DRIS para dois nutrientes $(\mathrm{A}$ e $\mathrm{B})$ quaisquer; $\mathrm{B}_{1}, \mathrm{~B}_{2}, \mathrm{~B}_{\mathrm{n}}$ correspondem aos nutrientes relacionados ao nutriente $A$; e $n$ corresponde ao número de nutrientes analisados.

As fórmulas DRIS, para cálculo dos índices, foram transformadas logaritmicamente. $\mathrm{O}$ uso de relações bivariadas transformadas logaritmicamente foi proposto para corrigir os desvios observados na distribuição normal entre as formas diretas e inversas de expressão de uma mesma relação (Beverly, 1987). $\mathrm{Na}$ verdade, para quaisquer dois nutrientes $\mathrm{R}$ e $\mathrm{S}$, cujas concentrações médias são expressas em [r] e [s], em que $\mathrm{R}>\mathrm{S}$, observa-se constantemente que $[\mathrm{r}] /[\mathrm{s}]>1 /[\mathrm{r}] /[\mathrm{s}]$. Este comportamento consiste em uma fraqueza na distribuição dos valores das relações bivariadas, uma vez que formas alternativas de expressão deveriam resultar em medidas equivalentes para o balanço entre os nutrientes. A falha introduz erros sistemáticos na abordagem tradicional. Todavia, com a transformação logarítmica - por definição: log $[\mathrm{r}] /[\mathrm{s}]=-\log 1 /([\mathrm{r}] /[\mathrm{s}]-$ ambas as formas de expressão resultam em desvios de mesma magnitude. Assim, foi feita a transformação logarítmica em todas as relações bivariadas utilizadas nas fórmulas de Jones (1981) e Wadt et al. (2007). A fórmula de Parent \& Dafir (1992) adota, originalmente, a transformação logarítmica.

Para a fórmula de Wadt et al. (2007), os valores para o coeficiente de sensibilidade (fk) foram 2,0, 2,0, 2,0, $0,5,0,5,1,5,1,5,1,0,0,5$ e 0,5 , respectivamente para $\mathrm{N}, \mathrm{P}, \mathrm{K}, \mathrm{Ca}, \mathrm{Mg}, \mathrm{Zn}, \mathrm{B}, \mathrm{Fe}, \mathrm{Mn}$ e $\mathrm{Cu}$. Na fórmula de Wadt et al. (2007), foi também adotada a correção usada para macronutrientes de resposta rara $(\mathrm{Ca}$ e $\mathrm{Mg})$. Esta correção fez-se necessária porque, quando o valor da relação se afastava de zero para valores negativos $[(\mathrm{A} / \mathrm{B}) \leq(\mathrm{a} / \mathrm{b}) / 2]$, o valor do módulo da função DRIS, que deveria aumentar, passava a diminuir. Esta constatação foi apresentada por Wadt \& Lemos (2010) na correção da respectiva fórmula. As equações, neste caso, foram modificadas para as expressões: quando $\log (\mathrm{A} / \mathrm{B})<\log (\mathrm{a} / \mathrm{b})$ e $\log (\mathrm{A} / \mathrm{B})>\log (\mathrm{a} / \mathrm{b}) / 2$, utiliza-se a função DRIS com sua expressão $\mathrm{f}(\mathrm{A} / \mathrm{B})=\mathrm{fk} \times\left\{[\log (\mathrm{A} / \mathrm{B})-\log (\mathrm{a} / \mathrm{b})] / \sigma_{\log (\mathrm{a} b)}\right\} \times[\log (\mathrm{A} / \mathrm{B}) /$ $\log (\mathrm{a} / \mathrm{b})]$. Entretanto, quando $\log (\mathrm{A} / \mathrm{B})<\log (\mathrm{a} / \mathrm{b})$ e $\log (\mathrm{A} / \mathrm{B}) \leq \log (\mathrm{a} / \mathrm{b}) / 2$, a função DRIS assume a forma $\mathrm{f}(\mathrm{A} / \mathrm{B})=\mathrm{fk} \times\left\{[\log (\mathrm{A} / \mathrm{B})-\log (\mathrm{a} / \mathrm{b})] / \sigma_{\log (\mathrm{a} / \mathrm{b})}\right\}$. Quando $\log (\mathrm{A} / \mathrm{B}) \geq \log (\mathrm{a} / \mathrm{b})$, a função DRIS assume a forma: $\mathrm{f}(\mathrm{A} / \mathrm{B})=\mathrm{fk} \times\left\{[\log (\mathrm{A} / \mathrm{B})-\log (\mathrm{a} / \mathrm{b})] / \sigma_{\log (\mathrm{a} / \mathrm{b})}\right\} \times[\log (\mathrm{a} / \mathrm{b}) /$ $\log (\mathrm{A} / \mathrm{B})]$. Nessas expressões, tem-se que: $\log (\mathrm{A} / \mathrm{B})$ corresponde à relação entre os nutrientes $\mathrm{A}$ e $\mathrm{B}$ na amostra; $\log (\mathrm{a} / \mathrm{b})$ corresponde à relação entre os nutrientes A e B na norma de referência; e $\sigma_{\log (a b)}$ corresponde ao desvio-padrão da relação A e B na norma de referência.

A interpretação dos índices DRIS, obtidos pelas fórmulas simplificada, multivariada e da modelagem das funções, foi realizada com base no critério do potencial de resposta à adubação (PRA) (Wadt, 2005). Adotaram-se três categorias para o estado nutricional, com base no valor do índice DRIS (In) em módulo comparado ao índice de balanço nutricional médio (IBNm): equilibrado, quando $|\mathrm{In}|<\mathrm{IBNm}$; insuficiente, quando $|\mathrm{In}|>\mathrm{IBNm}, \quad \mathrm{In}<0$; e em excesso, quando $|\mathrm{In}|>\mathrm{IBNm}$ e In $>0$. O IBNm foi calculado como a média aritmética do módulo dos índices DRIS de cada amostra avaliada.

Para a determinação do estado nutricional das mangueiras, adotaram-se os limites da faixa de suficiência sugeridos por Quaggio (1996): N, 12 a $14 \mathrm{~g} \mathrm{~kg}^{-1}$; P, 0,8 a 1,6 g kg-1; K, 5 a $10 \mathrm{~g} \mathrm{~kg}^{-1}$; Ca, 20 a $35 \mathrm{~g} \mathrm{~kg}^{-1} ; \mathrm{Mg}, 2,5$ a $5,0 \mathrm{~g} \mathrm{~kg}^{-1}$; B, 50 a $100 \mathrm{mg} \mathrm{kg}^{-1}$; $\mathrm{Zn}, 20$ a $40 \mathrm{mg} \mathrm{kg}^{-1}$; Fe, 50 a $200 \mathrm{mg} \mathrm{kg}^{-1}$; Mn, 50 a $100 \mathrm{mg} \mathrm{kg}^{-1}$; Cu, 10 a $50 \mathrm{mg} \mathrm{kg}^{-1}$. Estes valores foram adotados por serem os adotados rotineiramente na região do presente estudo.

Para cada nutriente, as amostras foram classificadas pelo método das faixas de suficiência como: deficientes, se o teor do nutriente fosse menor que o limite inferior da faixa de suficiência; normais, se dentro da faixa; e em toxidez, se maior que o limite superior da faixa. A interpretação do estado nutricional, obtida por esse processo, foi considerada como se de fato representasse o verdadeiro estado nutricional das plantas, embora se reconheça a inexatidão inerente a essa interpretação, principalmente pela ocorrência de efeitos de diluição e concentração de nutrientes (Jarrel \& Beverly, 1981).

A seguir, para cada método, considerando-se o diagnóstico determinado pelo método da faixa de suficiência como o verdadeiro estado nutricional dos pomares, calcularam-se as diversas medidas de acurácia (Wadt \& Lemos, 2010) para cada fórmula DRIS. Como o objetivo do trabalho foi demonstrar 
como as medidas de acurácia descritas adiante podem ser aplicadas em diferentes fórmulas DRIS, e como estas mesmas medidas podem ser utilizadas, a partir de algoritmos definidos, para ajustar os coeficientes das funções DRIS e aumentar o número de diagnósticos corretos, a adoção do método das faixas de suficiência atende unicamente ao propósito de proporcionar uma referência que estime o verdadeiro estado nutricional dos pomares. Alternativas poderiam ser adotadas como, por exemplo, acompanhar a resposta dos pomares à adição (ou remoção) de nutrientes no programa de adubações e, assim, determinar seu verdadeiro estado em relação a cada um dos nutrientes avaliados.

Para avaliar as medidas de acurácia, foram adotados os seguintes conceitos: quanto ao estado nutricional, os pomares podem apresentar-se no estado fisiológico de deficiência nutricional, suficiência nutricional ou toxicidade nutricional; quanto ao método de diagnose nutricional, os pomares podem ser considerados como estando em situação de insuficiência nutricional, equilíbrio nutricional e excesso nutricional (Tabela 1). Sete medidas de acurácia podem ser derivadas a partir da comparação dessas situações, todas na escala de 0 a 1 (Wadt \& Lemos, 2010).

A acurácia global (AG) é uma medida genérica para a utilidade dos processos de diagnóstico, representada pela média aritmética de diagnósticos corretos em relação a cada situação de estado nutricional possível para os pomares, por meio da expressão $\mathrm{AG}=\left(\mathrm{I}_{\mathrm{V}} / \sum \mathrm{D}+\mathrm{Eq}_{\mathrm{v}} / \sum \mathrm{S}+\mathrm{Ex}_{\mathrm{V}} / \sum \mathrm{T}\right) / 3$, cujas variáveis encontram-se na Tabela 1 . Sua principal vantagem é que indica o desempenho global do método de diagnóstico nutricional, o que permite comparar diferentes metodologias por meio de único indicador; todavia, alta acurácia global não representa, necessariamente, melhor desempenho para uma dada metodologia. A desvantagem desta medida é que ela não distingue os diagnósticos quanto ao acerto em identificar cada um dos possíveis estados nutricionais e, como o número de situações para o estado nutricional não é uniforme, uma elevada acurácia global pode não indicar corretamente a capacidade do método em diagnosticar de modo correto plantas nutricionalmente deficientes daquelas suficientes.

A acurácia para insuficiência (ACI) é outra das sete medidas possíveis. Ela mede o grau de diagnósticos verdadeiros de insuficiência nutricional. Essa medida é calculada pela expressão $\mathrm{ACI}=\left\{2+\left[\mathrm{I}_{\mathrm{V}} / \sum \mathrm{D}-\left(\mathrm{I}_{\mathrm{F}(\mathrm{S})} / \sum \mathrm{S}+\mathrm{I}_{\mathrm{F}(\mathrm{T})} / \sum \mathrm{T}\right)\right]\right\} / 3$.

A acurácia quanto ao equilíbrio nutricional (ACEq) é uma medida do grau de diagnósticos verdadeiros de equilíbrio nutricional. Ela é calculada pela expressão $\mathrm{ACEq}=\left\{2+\left[\mathrm{Eq}_{\mathrm{V}} / \sum \mathrm{S}-\left(\mathrm{Eq}_{\mathrm{F}(\mathrm{D})} / \sum \mathrm{D}+\mathrm{Eq}_{\mathrm{F}(\mathrm{T})} / \sum \mathrm{T}\right)\right]\right\} / 3$.

A acurácia para excesso nutricional (ACEx) é uma medida do grau de diagnósticos verdadeiros para excesso nutricional. É calculada pela expressão $\mathrm{ACEx}=\left\{2+\left[\mathrm{Ex}_{\mathrm{V}} / \sum \mathrm{T}-\left(\mathrm{Ex}_{\mathrm{F}(\mathrm{S})} / \sum \mathrm{S}+\mathrm{Ex}_{\mathrm{F}(\mathrm{D})} / \sum \mathrm{D}\right)\right]\right\} / 3$.

A acurácia para deficiência nutricional (ACD) é uma medida do grau de acerto para os diagnósticos de deficiência nutricional. É calculada pela expressão $\mathrm{ACD}=\left\{2+\left[\mathrm{I}_{\mathrm{V}} / \sum \mathrm{I}-\left(\mathrm{Eq}_{\mathrm{F}(\mathrm{D})} / \sum \mathrm{Eq}+\mathrm{Ex}_{\mathrm{F}(\mathrm{D})} / \sum \mathrm{Ex}\right)\right]\right\} / 3$.

A acurácia para suficiência (ACS) é uma medida do grau de acerto para os diagnósticos de

Tabela 1. Distribuição dos casos de estado nutricional fisiológico, determinado experimentalmente ou indiretamente, e de diagnósticos produzidos por métodos de avaliação do estado nutricional das plantas.

\begin{tabular}{|c|c|c|c|c|}
\hline \multirow[t]{2}{*}{ Diagnóstico nutricional } & \multicolumn{3}{|c|}{ Estado nutricional fisiológico ${ }^{(1)}$} & \multirow[t]{2}{*}{ Subtotal } \\
\hline & Deficiência & Suficiência & Toxicidade & \\
\hline Insuficiência & $\mathrm{I}_{\mathrm{V}}$ & $\mathrm{I}_{\mathrm{F}(\mathrm{S})}$ & $\mathrm{I}_{\mathrm{F}(\mathrm{T})}$ & $\sum \mathrm{I}$ \\
\hline Equilíbrio & $\mathrm{Eq}_{\mathrm{F}(\mathrm{D})}$ & $\mathrm{Eq}_{\mathrm{v}}$ & $\mathrm{Eq}_{\mathrm{F}(\mathrm{T})}$ & $\sum \mathrm{Eq}$ \\
\hline$\underline{\text { Excesso }}$ & $\mathrm{Ex}_{\mathrm{F}(\mathrm{D})}$ & $\mathrm{Ex}_{\mathrm{F}(\mathrm{S})}$ & $\mathrm{Ex}_{\mathrm{V}}$ & $\sum \mathrm{Ex}$ \\
\hline Subtotal & $\sum \mathrm{D}$ & $\sum \mathrm{S}$ & $\sum \mathrm{T}$ & $\sum \sum \mathrm{n}$ \\
\hline
\end{tabular}

${ }^{(1)} \mathrm{I}_{\mathrm{V}}$, contagem do número de diagnósticos verdadeiros para insuficiência nutricional; $\mathrm{I}_{\mathrm{F}(\mathrm{S})}$, contagem do número de diagnósticos falsos para insuficiência, em situações de reconhecida suficiência nutricional; $\mathrm{I}_{\mathrm{F}(\mathrm{T})}$, contagem do número de diagnósticos falsos para insuficiência, em situações de reconhecida toxicidade nutricional; $\mathrm{Eq}_{\mathrm{V}}$, contagem do número de diagnósticos verdadeiros quanto ao equilíbrio nutricional; $\mathrm{Eq}_{\mathrm{F}(\mathrm{D})}$ ), contagem do número de diagnósticos falsos quanto ao equilíbrio, em situações de reconhecida deficiência nutricional; $\mathrm{Eq}_{\mathrm{F}(\mathrm{T})}$, contagem do número de diagnósticos falsos quanto ao equilíbrio, em situações de reconhecida toxicidade nutricional; $\mathrm{Ex}_{\mathrm{V}}$, contagem do número de diagnósticos verdadeiros para excesso nutricional; $\mathrm{Ex}_{\mathrm{F}(\mathrm{D})}$, contagem do número de diagnósticos falsos para excesso, em situações de reconhecida deficiência nutricional; $\mathrm{Ex}_{\mathrm{F}(\mathrm{S})}$, contagem do número de diagnósticos falsos para excesso, em situações de reconhecida suficiência nutricional; $\sum \mathrm{D}$, total de casos de deficiência nutricional; $\sum \mathrm{S}$, total de casos de suficiência nutricional; $\sum \mathrm{T}$, total de casos de toxicidade nutricional; $\sum \mathrm{I}$, total de diagnósticos de insuficiência nutricional; $\sum \mathrm{Eq}$, total de diagnósticos de equilíbrio nutricional; $\sum$ Ex, total de diagnósticos de excesso nutricional; $\sum \sum \mathrm{n}$, total de casos computados. 
suficiência nutricional. É calculada pela expressão $\mathrm{ECS}=\left\{2+\left[\mathrm{Eq}_{\mathrm{V}} / \sum \mathrm{Eq}-\left[\mathrm{I}_{\mathrm{F}(\mathrm{S})} / \sum \mathrm{I}+\mathrm{Ex}_{\mathrm{F}(\mathrm{S})} / \sum \mathrm{Ex}\right)\right]\right\} / 3$.

Finalmente, a acurácia para toxicidade nutricional (ACT) é uma medida do grau de acerto para os diagnósticos de toxicidade nutricional. É calculada pela expressão $\mathrm{ACT}=\left\{2+\left[\mathrm{Ex}_{\mathrm{V}} / \sum \mathrm{Ex}-\left(\mathrm{I}_{\mathrm{F}(\mathrm{T})} / \sum \mathrm{I}+\mathrm{Eq}_{\mathrm{F}(\mathrm{T})} / \sum \mathrm{Eq}\right)\right]\right\} / 3$.

Medidas de acurácia elevadas (próximo de 1) significam que a maioria dos diagnósticos do estado nutricional da planta foram corretos, e medidas baixas (próximo de 0 ) indicam que houve muitos diagnósticos falsos.

Exclusivamente para a fórmula de Wadt et al. (2007), os valores dos coeficientes de sensibilidade (fator k) de cada função DRIS foram modelados para aumentar a utilidade dos diagnósticos produzidos (Wadt \& Lemos, 2010). A modelagem consiste de um processo iterativo para o cálculo da acurácia a partir de um conjunto de diagnósticos obtidos. Em seguida, do incremento ou decremento em $1 \%$ no valor do coeficiente de sensibilidade de cada função DRIS (Tabela 2), é realizado um novo conjunto de diagnósticos. Novamente, no cálculo de novas medidas de acurácia, o ciclo é repetido até um limite pré-definido de iterações. Em cada ciclo, a avaliação da acurácia de determinado conjunto de diagnósticos resulta em alterações nos coeficientes de ajustes das funções DRIS, o que, por sua vez, proporcionará um novo conjunto de diagnósticos e, novamente, novas medidas de acurácia. As modificações feitas nos coeficientes de ajuste de cada função
DRIS objetivam melhorar determinada medida de acurácia, priorizando-se as medidas de acurácia para deficiência e insuficiência. No presente trabalho, foram testadas 50, 100 e 150 iterações.

Os cálculos relacionados à obtenção das normas DRIS, dos índices DRIS, dos critérios de interpretação dos índices DRIS, das medidas de acurácia e da modelagem das funções DRIS pelo processo iterativo foram realizados por meio do programa DRIS Manga (DRIS, 2010).

\section{Resultados e Discussão}

Neste trabalho, o método da faixa de suficiência foi adotado como referência para estimar o verdadeiro estado nutricional dos pomares; reconhece-se, todavia, que esta situação não é a mais adequada, já que o próprio método das faixas de suficiência poderia ter sua utilidade para os diagnósticos nutricionais avaliados por meio das medidas de acurácia propostas. Entretanto, ao se adotar este procedimento, foi possível estimar o provável estado nutricional das plantas quanto a cada nutriente, o que permitiu a comparação entre fórmulas DRIS e a modelagem das funções DRIS, com base nas medidas de acurácia dos diagnósticos.

A acurácia global dos diagnósticos, realizados pela fórmula de Jones (1981), foi acima de $80 \%$ para $\mathrm{P}, \mathrm{B}$ e Fe, abaixo de $60 \%$ para $\mathrm{N}$ e $\mathrm{Mn}$ e ficou entre 60 e $75 \%$ para os demais nutrientes (Tabela 3). Entretanto, se observada a acurácia para deficiência, esta fórmula apresentou um bom desempenho, com valores sempre

Tabela 2. Ajustes no fator k, para a modelagem das funções DRIS, em função da acurácia dos diagnósticos alcançados pela interpretação dos índices DRIS pelo método do potencial de resposta à adubação.

\begin{tabular}{|c|c|c|}
\hline Situação $^{(1)}$ & Ajuste a ser realizado no fator $\mathrm{k}$ & Problema relacionado ao manejo da adubação \\
\hline $\mathrm{ACD}<0,7$ e $\mathrm{ACD}<\mathrm{ACI}$ & Aumentar $1 \%$ & $\begin{array}{l}\text { Grande número de diagnósticos falsos para deficiência, o que resultaria em adubação menor } \\
\text { do que a realmente necessária. }\end{array}$ \\
\hline $\mathrm{ACI}<0,7$ e $\mathrm{ACI}<\mathrm{ACD}$ & Diminuir $1 \%$ & $\begin{array}{l}\text { Grande número de diagnósticos falsos para insuficiência, o que resultaria em adubação } \\
\text { desnecessária. }\end{array}$ \\
\hline $\mathrm{ACEq}<0,5$ e $\mathrm{ACEq}<\mathrm{ACS}$ & Aumentar $1 \%$ & $\begin{array}{l}\text { Grande número de diagnósticos falsos quanto ao equilíbrio nutricional, o que implicaria } \\
\text { a possibilidade de perda de rendimento, em razão tanto da falta quanto do excesso de } \\
\text { adubação. }\end{array}$ \\
\hline $\mathrm{ACS}<0,5$ e $\mathrm{ACS}<\mathrm{ACEq}$ & Diminuir 1\% & $\begin{array}{l}\text { Grande número de diagnósticos falsos para suficiência, o que implicaria a possibilidade de } \\
\text { perda de rendimento em razão tanto da falta quanto do excesso de adubação. }\end{array}$ \\
\hline $\mathrm{ACEx}<0,4$ e $\mathrm{ACEx}<\mathrm{ACT}$ & Diminuir $1 \%$ & $\begin{array}{l}\text { Grande número de diagnósticos falsos para excesso, o que resultaria em aumento de nutrientes } \\
\text { já fornecidos, em quantidade excessiva. }\end{array}$ \\
\hline $\mathrm{ACT}<0,4$ e $\mathrm{ACT}<\mathrm{ACEx}$ & Aumentar $1 \%$ & $\begin{array}{l}\text { Grande número de diagnósticos falsos para toxicidade, o que resultaria em diminuição dos } \\
\text { nutrientes fornecidos em quantidades adequadas ou equilibradas. }\end{array}$ \\
\hline
\end{tabular}

Nenhuma das anteriores $\quad$ Manter o mesmo valor

${ }^{(1)} \mathrm{ACD}$, acurácia de deficiência; ACI, acurácia de insuficiência; ACEq, acurácia de equilíbrio; ACS, acurácia de suficiência; ACEx, acurácia de excesso; $\mathrm{ACT}$, acurácia de toxicidade. 
superiores a $67 \%$, e acima de $85 \%$ para $\mathrm{Ca}, \mathrm{Mg}$ e $\mathrm{B}$. $\mathrm{Na}$ acurácia por insuficiência, o desempenho para todos os nutrientes foi ainda maior. Esses resultados indicam que, tomando-se o método das faixas de suficiência proposto por Quaggio et al. (1996) como referência, a fórmula de Jones (1981) possui elevada utilidade, já que resultou em baixo número de diagnósticos falsos para insuficiência e deficiência. Neste caso, considerando-se que os pomares foram adubados durante o ciclo de produção, as medidas de acurácia indicam que o manejo destas adubações proporcionou uma nutrição equilibrada. As demais medidas de acurácia, menos relevantes no manejo da adubação, apresentaram desempenho, em sua maioria, superior a $50 \%$ (Tabela 3 ).

Com o uso da fórmula para relações multivariadas (Parent \& Dafir, 1992), a acurácia global também foi superior a $80 \%$ para $\mathrm{P}, \mathrm{K}$ e Fe, porém, para $\mathrm{Mn}$ ficou abaixo de $60 \%$. Ao se avaliar a acurácia para deficiência e insuficiência, observa-se que o desempenho dessa fórmula foi muito semelhante ao da fórmula de Jones (1981), embora para Ca e B a acurácia para deficiência tenha sido superior a $85 \%$, com acurácia para os demais nutrientes superior a $67 \%$. A acurácia para insuficiência

Tabela 3. Valores de acurácia global (G), de deficiência (D), de suficiência (S), de toxicidade (T), de insuficiência (I), de equilíbrio (Eq), e de excesso (Exc), para diagnósticos nutricionais de mangueiras cultivadas no perímetro irrigado do Semiárido nordestino, obtidos pelo método DRIS, com uso da fórmula simplificada de Jones (1981), da fórmula de relações multivariadas de Parent \& Dafir (1992) e da fórmula de modelagem das funções DRIS de Wadt et al. (2007), tendo-se considerado como referência o estado nutricional pelo método das faixas de suficiência proposto por Quaggio (1996).

\begin{tabular}{|c|c|c|c|c|c|c|c|}
\hline Nutriente & G & $\mathrm{D}$ & $\mathrm{S}$ & $\mathrm{T}$ & I & $\mathrm{Eq}$ & Exc \\
\hline & \multicolumn{7}{|c|}{ Fórmula simplificada de Jones (1981) } \\
\hline $\mathrm{N}$ & 0,55 & 0,75 & 0,57 & 0,60 & 0,79 & 0,58 & 0,74 \\
\hline $\mathrm{P}$ & 0,89 & 0,67 & 0,45 & 0,78 & 0,94 & 0,89 & 0,95 \\
\hline $\mathrm{K}$ & 0,76 & 0,67 & 0,50 & 0,84 & 0,94 & 0,76 & 0,82 \\
\hline $\mathrm{Ca}$ & 0,70 & 0,87 & 0,56 & 0,69 & 0,80 & 0,70 & 0,89 \\
\hline $\mathrm{Mg}$ & 0,68 & 0,85 & 0,52 & 0,67 & 0,77 & 0,68 & 0,91 \\
\hline B & 0,88 & 0,97 & 0,86 & 0,89 & 0,98 & 0,88 & 0,90 \\
\hline $\mathrm{Zn}$ & 0,72 & 0,80 & 0,66 & 0,87 & 0,87 & 0,72 & 0,86 \\
\hline $\mathrm{Fe}$ & 0,86 & 0,72 & 0,54 & 0,82 & 0,92 & 0,86 & 0,93 \\
\hline $\mathrm{Mn}$ & 0,44 & 0,68 & 0,54 & 0,47 & 0,63 & 0,48 & 0,77 \\
\hline \multirow[t]{2}{*}{$\mathrm{Cu}$} & 0,69 & 0,67 & 0,56 & 0,89 & 0,76 & 0,69 & 0,94 \\
\hline & \multicolumn{7}{|c|}{ Fórmula de relações multivariadas de Parent \& Dafir (1992) } \\
\hline $\mathrm{N}$ & 0,60 & 0,73 & 0,60 & 0,55 & 0,78 & 0,65 & 0,77 \\
\hline $\mathrm{P}$ & 0,85 & 0,67 & 0,42 & 0,75 & 0,93 & 0,85 & 0,92 \\
\hline $\mathrm{K}$ & 0,74 & 0,67 & 0,47 & 0,81 & 0,90 & 0,74 & 0,84 \\
\hline $\mathrm{Ca}$ & 0,68 & 0,86 & 0,55 & 0,69 & 0,81 & 0,68 & 0,87 \\
\hline $\mathrm{Mg}$ & 0,63 & 0,78 & 0,46 & 0,67 & 0,77 & 0,64 & 0,86 \\
\hline B & 0,83 & 0,97 & 0,86 & 0,89 & 0,94 & 0,83 & 0,88 \\
\hline $\mathrm{Zn}$ & 0,74 & 0,82 & 0,70 & 0,88 & 0,89 & 0,74 & 0,85 \\
\hline $\mathrm{Fe}$ & 0,80 & 0,71 & 0,49 & 0,78 & 0,92 & 0,80 & 0,88 \\
\hline $\mathrm{Mn}$ & 0,41 & 0,68 & 0,52 & 0,49 & 0,64 & 0,44 & 0,74 \\
\hline \multirow[t]{2}{*}{$\mathrm{Cu}$} & 0,74 & 0,67 & 0,58 & 0,91 & 0,82 & 0,74 & 0,92 \\
\hline & \multicolumn{7}{|c|}{ Fórmula de modelagem das funções DRIS de Wadt et al. (2007) } \\
\hline $\mathrm{N}$ & 0,37 & 0,69 & 0,59 & 0,45 & 0,50 & 0,55 & 0,69 \\
\hline $\mathrm{P}$ & 0,67 & 1,00 & 0,69 & 0,69 & 1,00 & 0,67 & 0,67 \\
\hline $\mathrm{K}$ & 0,75 & 0,67 & 0,47 & 0,80 & 0,96 & 0,75 & 0,79 \\
\hline $\mathrm{Ca}$ & 0,33 & 0,86 & 0,86 & 1,00 & 0,67 & 0,33 & 0,67 \\
\hline $\mathrm{Mg}$ & 0,66 & 0,88 & 0,55 & 0,67 & 0,67 & 0,66 & 1,00 \\
\hline B & 0,48 & 0,87 & 0,77 & 0,90 & 0,80 & 0,48 & 0,68 \\
\hline $\mathrm{Zn}$ & 0,52 & 0,75 & 0,67 & 0,76 & 0,76 & 0,58 & 0,69 \\
\hline $\mathrm{Fe}$ & 0,45 & 0,68 & 0,62 & 0,93 & 0,78 & 0,45 & 0,67 \\
\hline $\mathrm{Mn}$ & 0,33 & 0,68 & 0,57 & 0,44 & 0,61 & 0,39 & 0,67 \\
\hline $\mathrm{Cu}$ & 0,67 & 1,00 & 0,86 & 0,86 & 1,00 & 0,67 & 0,67 \\
\hline
\end{tabular}


foi superior a $85 \%$ para $\mathrm{P}, \mathrm{K}, \mathrm{B}, \mathrm{Zn}$ e Fe e acima de $67 \%$ para os demais nutrientes (Tabela 3 ).

Da comparação das duas fórmulas, deduz-se que ambas apresentaram comportamentos semelhantes, com uma pequena superioridade para a fórmula de Jones (1981). Deve-se considerar, no entanto, que a fórmula de Parent \& Dafir (1992) tem como vantagem a facilidade dos cálculos e o fato de as variáveis serem adequadas para estudos com métodos estatísticos avançados (Parent \& Natale, 2008).

A fórmula de Wadt et al. (2007) apresentou comportamento muito inferior às demais fórmulas testadas, para a acurácia global, em que somente $\mathrm{K}$ atingiu o patamar de $75 \%$. Entretanto, se forem consideradas as acurácias para deficiência e insuficiência, o cenário se reverte. A acurácia para deficiência foi superior a $85 \%$ para $\mathrm{P}, \mathrm{Ca}, \mathrm{Mg}, \mathrm{B}, \mathrm{Cu}$ e não foi abaixo de $67 \%$ para nenhum nutriente. A acurácia para insuficiência foi superior a $85 \%$ para $\mathrm{P}, \mathrm{K}, \mathrm{Cu}$ e inferior a $67 \%$ somente para $\mathrm{N}$ e Mn (Tabela 3). Portanto, a fórmula de Wadt et al. (2007) teve pior desempenho para acurácia global, mas o melhor desempenho para as medidas de acurácia mais relevantes para o manejo das adubações (acurácia para deficiência e insuficiência).

Tabela 4. Valores de acurácia global (G), de deficiência (D), de suficiência (S), de toxicidade (T), de insuficiência (I), de equilíbrio (Eq), de excesso (Exc), e fator de ajuste das funções DRIS (fk), para diagnósticos nutricionais de mangueiras cultivadas no perímetro irrigado do Semiárido nordestino, com uso da fórmula de Wadt et al. (2007), após 50, 100 e 150 iterações, tendo-se considerado, como referência, o estado nutricional determinado pelo método das faixas de suficiência proposto por Quaggio (1996).

\begin{tabular}{|c|c|c|c|c|c|c|c|c|}
\hline Nutriente & $\mathrm{G}$ & $\mathrm{D}$ & $\mathrm{S}$ & $\mathrm{T}$ & $\mathrm{I}$ & $\mathrm{Eq}$ & Exc & $\mathrm{fk}$ \\
\hline & \multicolumn{8}{|c|}{ Após 50 iterações } \\
\hline $\mathrm{N}$ & 0,36 & 0,69 & 0,59 & 0,51 & 0,58 & 0,45 & 0,69 & 1,20 \\
\hline $\mathrm{P}$ & 0,67 & 1,00 & 0,69 & 0,69 & 1,00 & 0,67 & 0,67 & 2,00 \\
\hline $\mathrm{K}$ & 0,77 & 0,67 & 0,48 & 0,81 & 0,93 & 0,77 & 0,84 & 3,32 \\
\hline $\mathrm{Ca}$ & 0,33 & 0,86 & 0,86 & 1,00 & 0,67 & 0,33 & 0,67 & 0,30 \\
\hline $\mathrm{Mg}$ & 0,67 & 0,89 & 0,89 & 1,00 & 0,67 & 0,67 & 1,00 & 0,30 \\
\hline B & 0,51 & 0,83 & 0,71 & 0,89 & 0,82 & 0,51 & 0,69 & 1,81 \\
\hline $\mathrm{Zn}$ & 0,51 & 0,75 & 0,65 & 0,76 & 0,76 & 0,57 & 0,69 & 1,50 \\
\hline $\mathrm{Fe}$ & 0,43 & 0,68 & 0,61 & 0,90 & 0,69 & 0,51 & 0,67 & 1,26 \\
\hline $\mathrm{Mn}$ & 0,40 & 0,69 & 0,52 & 0,51 & 0,71 & 0,42 & 0,67 & 1,22 \\
\hline \multirow[t]{2}{*}{$\mathrm{Cu}$} & 0,67 & 1,00 & 0,86 & 0,86 & 1,00 & 0,67 & 0,67 & 0,50 \\
\hline & \multicolumn{8}{|c|}{ Após 100 iterações } \\
\hline $\mathrm{N}$ & 0,34 & 0,68 & 0,62 & 0,53 & 0,64 & 0,36 & 0,68 & 0,72 \\
\hline $\mathrm{P}$ & 0,67 & 1,00 & 0,69 & 0,69 & 1,00 & 0,67 & 0,67 & 2,00 \\
\hline $\mathrm{K}$ & 0,72 & 0,67 & 0,46 & 0,78 & 0,87 & 0,73 & 0,84 & 5,51 \\
\hline $\mathrm{Ca}$ & 0,33 & 0,86 & 0,86 & 1,00 & 0,67 & 0,33 & 0,67 & 0,18 \\
\hline $\mathrm{Mg}$ & 0,67 & 0,89 & 0,89 & 1,00 & 0,67 & 0,67 & 1,00 & 0,18 \\
\hline B & 0,49 & 0,78 & 0,69 & 0,87 & 0,77 & 0,50 & 0,70 & 2,00 \\
\hline $\mathrm{Zn}$ & 0,51 & 0,75 & 0,65 & 0,76 & 0,75 & 0,57 & 0,69 & 1,50 \\
\hline $\mathrm{Fe}$ & 0,43 & 0,68 & 0,60 & 0,90 & 0,71 & 0,49 & 0,67 & 1,25 \\
\hline $\mathrm{Mn}$ & 0,37 & 0,69 & 0,49 & 0,53 & 0,68 & 0,38 & 0,67 & 1,14 \\
\hline \multirow[t]{2}{*}{$\mathrm{Cu}$} & 0,67 & 1,00 & 0,86 & 0,86 & 1,00 & 0,67 & 0,67 & 0,50 \\
\hline & \multicolumn{8}{|c|}{ Após 150 iterações } \\
\hline $\mathrm{N}$ & 0,33 & 0,65 & 0,64 & 0,49 & 0,64 & 0,34 & 0,68 & 0,44 \\
\hline $\mathrm{P}$ & 0,67 & 1,00 & 0,69 & 0,69 & 1,00 & 0,67 & 0,67 & 2,00 \\
\hline $\mathrm{K}$ & 0,71 & 0,67 & 0,46 & 0,78 & 0,85 & 0,72 & 0,85 & 9,15 \\
\hline $\mathrm{Ca}$ & 0,33 & 0,86 & 0,86 & 1,00 & 0,67 & 0,33 & 0,67 & 0,11 \\
\hline $\mathrm{Mg}$ & 0,67 & 0,89 & 0,89 & 1,00 & 0,67 & 0,67 & 1,00 & 0,11 \\
\hline B & 0,48 & 0,78 & 0,66 & 0,87 & 0,77 & 0,49 & 0,70 & 2,39 \\
\hline $\mathrm{Zn}$ & 0,48 & 0,74 & 0,64 & 0,77 & 0,74 & 0,52 & 0,69 & 1,50 \\
\hline $\mathrm{Fe}$ & 0,44 & 0,68 & 0,63 & 0,91 & 0,68 & 0,54 & 0,67 & 1,34 \\
\hline $\mathrm{Mn}$ & 0,40 & 0,69 & 0,52 & 0,51 & 0,71 & 0,42 & 0,67 & 1,25 \\
\hline $\mathrm{Cu}$ & 0,67 & 1,00 & 0,86 & 0,86 & 1,00 & 0,67 & 0,67 & 0,50 \\
\hline
\end{tabular}


A vantagem desta fórmula é a possibilidade de se modelar os diagnósticos com interpretações mais precisas (Wadt et al., 2007; Wadt \& Lemos, 2010). Se verificar o desempenho desta fórmula após 50, 100 e 150 iterações (Tabela 4), observa-se que a acurácia para deficiência foi sempre superior a $85 \%$, para os nutrientes $\mathrm{P}, \mathrm{Ca}, \mathrm{Mg}, \mathrm{Cu}$, e que apenas $\mathrm{B}$ diminuiu o valor da acurácia para abaixo deste limite após 100 e 150 iterações. Em todas as iterações, nenhum nutriente apresentou acurácia para deficiência inferior a $67 \%$, exceto o $\mathrm{N}$ com modelagem para 150 iterações. Com relação à acurácia para insuficiência, também em todas as iterações testadas, $\mathrm{P}, \mathrm{K}$ e $\mathrm{Cu}$ tiveram o valor da acurácia superior a $85 \%$, e $\mathrm{B}$, somente na modelagem com 50 iterações. À exceção de $\mathrm{N}$, nenhum outro nutriente apresentou acurácia para insuficiência inferior a $67 \%$.

Embora a modelagem das fórmulas DRIS tenha resultado em uma perda da acurácia de deficiência e de insuficiência para $\mathrm{B}$, houve um considerável ganho no diagnóstico de $\mathrm{N}$, cuja acurácia para insuficiência passou de $50 \%$ (Tabela 3 ) para $64 \%$ (Tabela 4), o que foi acompanhado de pequena perda da acurácia por deficiência, que passou de 69\% (Tabela 3) para 65\% (Tabela 4), o que demonstra o potencial da modelagem para a melhoria da utilidade dos diagnósticos nutricionais.

Comparando-se os métodos de Jones (1981) (Tabela 3) e a modelagem das funções DRIS após 150 iterações (Tabela 4), nota-se que apresentam diferenças em suas utilidades, se forem considerados os principais macro e micronutrientes, conforme discussão a seguir. Para a acurácia de deficiência e insuficiência, o método de Jones (1981) foi superior para $\mathrm{N}$ (75x65\% e $79 \times 64 \%$, respectivamente), inferior para $\mathrm{P}(67 \times 100 \%$ e $94 \times 100 \%$, respectivamente) e superior para K (67x67\% e 94x85\%, respectivamente).

Para micronutrientes, também para a acurácia de deficiência e insuficiência, o método de Jones (1981) foi superior à modelagem das funções DRIS, após 150 iterações, para B $(97 \times 78 \%$ e 98x77\%, respectivamente), para $\mathrm{Zn}(80 \times 74 \%$ e $87 \times 74 \%$, respectivamente) e inferior para $\mathrm{Mn}(68 \times 69 \%$ e $63 \times 71 \%$, respectivamente).

No decorrer das sucessivas iterações para a modelagem das funções DRIS, os valores de fator de ajuste $\mathrm{k}$ foram se alterando, com tendência crescente de: diminuição do fator para $\mathrm{N}$ (de 2,0 para 0,44$), \mathrm{Ca}$ e $\mathrm{Mg}$ (de 0,5 para 0,11); aumento para $\mathrm{K}$ (de 2,0 para 9,15), Mn (de 0,50 para 1,14) e para Fe (de 1,0 para $1,34)$; e manutenção do valor inicial para $\mathrm{P}(2,0)$ e para B $(1,50)$ (Tabela 4).

Todavia, o monitoramento da resposta dos pomares aos ajustes nas recomendações dos nutrientes poderá resultar em outras combinações para esses coeficientes, de forma que não se recomenda, neste momento, a utilização desses valores, mas, sim, o processo de monitoramento nutricional que poderá conduzir a formulações mais adequadas de interpretação do estado nutricional. Esse procedimento difere do adotado por Bataglia et al. (2004) em cafeeiros, quando os valores para os coeficientes foram previamente definidos. Possivelmente, o fato de Bataglia et al. (2004) não terem obtido melhor utilidade para os diagnósticos produzidos tenha sido decorrente de não se ter permitido que o ajuste fosse feito iterativamente.

$\mathrm{Na}$ acurácia de toxicidade, o método de Jones (1981) mostrou-se equivalente ao método CND, e foi superior a este método e ao de Wadt et al. (2007) com 150 iterações, quanto à acurácia para excesso. O método de Jones (1981) também foi superior ao de Wadt et al. (2007) com 150 iterações quanto à acurácia para toxicidade (Tabelas 3 e 4). A modelagem das funções DRIS não se mostrou eficiente para melhorar a acurácia para toxicidade ou excesso (Tabelas 3 e 4), o que era de certo modo esperado, já que o algoritmo busca inicialmente maiores ganhos para as acurácia de deficiência e insuficiência (Tabela 2) (Wadt \& Lemos, 2010).

Com respeito às medidas de acurácia para suficiência e equilíbrio, o método de Jones (1981) mostrou-se superior aos demais, em todas as situações, mesmo ao de Wadt et al. (2007) após as 150 iterações; além disso, a modelagem das funções DRIS também não resultou em ganhos significativos, pelo mesmo motivo observado para acurácia por toxicidade e excesso.

Esses resultados indicam que a fórmula de Jones (1981) é a mais adequada, em situações em que a preocupação seja a de reduzir ou minimizar o uso de fertilizantes. Do ponto de vista ambiental, caso se busque eliminar o risco de adubações excessivas, a fórmula de Jones (1981), juntamente com a fórmula do método CND, é a mais indicada por resultar em maior acurácia nos diagnósticos de suficiência e excesso.

Por sua vez, a fórmula de Wadt et al. (2007), desde que modelada adequadamente, contribui para se evitarem falsos diagnósticos para deficiência 
- deixar de recomendar o nutriente quando ele é necessário e mesmo para diminuir falsos diagnósticos de insuficiência - recomendação do nutriente quando ele não é necessário -, sendo, portanto, indicada para situações em que se almeja alcançar o potencial produtivo do pomar.

As medidas de acurácia mostram-se promissoras como alternativa para mensurar a utilidade dos diagnósticos nutricionais, porém, há necessidade de validações mais rigorosas para que sejam adotadas em sistemas de monitoramento nutricional de pomares comerciais.

\section{Conclusões}

1. As fórmulas simplificadas de Jones, para relações bivariadas, e a fórmula para relações multivariadas de Parent \& Dafir, apresentam desempenho semelhante e devem ser igualmente recomendadas para situações em que se pretende minimizar o uso de fertilizantes.

2. O uso da fórmula de Wadt et al. depende da aplicação do processo iterativo de modelagem (ajustes dos coeficientes das funções DRIS) e deve ser recomendada somente após a avaliação de sua utilidade com base nas medidas de acurácia.

3. A modelagem das funções DRIS contribui para melhorar a utilidade dos diagnósticos, no que se refere à acurácia de deficiência e de insuficiência, sem efeito positivo sobre o desempenho das outras medidas de acurácia.

\section{Agradecimentos}

Ao Conselho Nacional de Desenvolvimento Científico e Tecnológico, pelo apoio financeiro.

\section{Referências}

BATAGLIA, O.C.; QUAGGIO, J.A.; SANTOS, W.R. dos; ABREU, M.F. de. Diagnose nutricional do cafeeiro pelo DRIS, variandose a constante de sensibilidade dos nutrientes de acordo com a intensidade e freqüência de resposta na produção. Bragantia, v.63, p.253-263, 2004.

BEVERLY, R.B. Comparison of DRIS and alternative nutrient diagnostic methods for soybean. Journal of Plant Nutrition, v.10, p.901-920, 1987.

BEVERLY, R.B.; HALLMARK, W.B. Prescient diagnostic analysis: a proposed new approach to evaluating plant nutrient diagnostic methods. Communications in Soil Science and Plant Analysis, v.23, p.2633-2640, 1992.
DRIS. Sistema Integrado de Diagnose e Recomendação. Manga. Disponível em: <http://www.dris.com.br/>. Acesso em: 16 set. 2010.

JARREL, W.M.; BEVERLY, R.B. The dilution effect in plant nutrition studies. Advances in Agronomy, v.34, p.197-224, 1981.

JONES, C.A. Proposed modifications of the diagnosis and recommendation integrated system (DRIS) for interpreting plant analyses. Communications in Soil Science and Plant Analysis, v.12, p.785-794, 1981.

PARENT, L.E.; DAFIR, M. A theoretical concept of compositional nutrient diagnosis. Journal of the American Society for Horticultural Science, v.117, p.239-242, 1992.

PARENT, L.E.; NATALE, W. CND: vantagens e benefícios para culturas de alta produtividade. In: PRADO, R. de M.; ROZANE, D.E.; VALE, D.W. do; CORREIA, M.A.R.; SOUZA, H.A. (Ed.). Nutrição de plantas: diagnose foliar em grandes culturas. Jaboticabal: Universidade Estadual Paulista, 2008. p.105-114.

PARTELLI, F.L.; VIEIRA, H.D.; MONNERAT, P.H.; VIANA, A.P. Comparação de dois métodos DRIS para o diagnóstico de deficiências nutricionais do cafeeiro. Pesquisa Agropecuária Brasileira, v.41, p.301-306, 2006.

PINTO, P.A. da C. Avaliação do estado nutricional da mangueira Tommy Atkins pelo DRIS e da qualidade pós-colheita de frutos no Submédio São Francisco. 2002. 124p. Tese (Doutorado) Universidade Federal de Viçosa, Viçosa.

QUAGGIO, J.A. Adubação e calagem para a mangueira e qualidade dos frutos. In: SÃO JOSÉ, A.R.; SOUZA, I.V.B.; MARTINS FILHO, J.; MORAIS, O.M. (Ed.) Manga: tecnologia de produção e mercado. Vitória da Conquista: UESB, 1996. p.106-135.

SILVA, D.J.; QUAGGIO, J.A.; PINTO, P.A. da C.; PINTO, A.C. de Q.; MAGALHÃES, A.F. de J. Nutrição e adubação. In: GENÚ, P.J. de C.; PINTO, A.C. de Q. (Ed.). A cultura da mangueira. Brasília: Embrapa Informação Tecnológica, 2002. p.191-221.

WADT, P.G.S. Relationships between soil class and nutritional status of coffee plantations. Revista Brasileira de Ciência do Solo, v.29, p.227-234, 2005.

WADT, P.G.S.; LEMOS, C.O. Medidas de acurácia para diagnósticos nutricionais e seu impacto no manejo das adubações. In: PRADO, R. de M.; CECÍLIO FILHO, A.B.; CORREIA, M.A.R.; PUGA, A.P. (Ed.). Nutrição de plantas: diagnose foliar em hortaliças. Jaboticabal: Universidade Estadual Paulista, 2010. p.213-236.

WADT, P.G.S.; NOVAIS, R.F. de. O monitoramento nutricional frente aos métodos diagnósticos no planejamento das adubações. In: SIMPÓSIO SOBRE MONITORAMENTO NUTRICIONAL PARA A RECOMENDAÇÃO DA ADUBAÇÃO DE CUlTURAS, 1999, Piracicaba. Anais. Piracicaba: Potafos, 1999. p.70-80.

WADT, P.G.S.; SILVA, D.J.; MAIA, C.E.; TOMÉ JÚNIOR, J.B.; PINTO, P.A. da C.; MACHADO, P.L.O. de A. Modelagem de funções no cálculo dos índices DRIS. Pesquisa Agropecuária Brasileira, v.42, p.57-64, 2007. 